\title{
Metastasized pancreatic neuroendocrine tumor in a teenage girl: a case report
}

\author{
Tina Tremmel ${ }^{1 *}$, Stefan Holland-Cunz ${ }^{2}$ and Patrick Günther ${ }^{1}$
}

\begin{abstract}
Introduction: Metastasized pancreatic neuroendocrine tumors are extremely rare malignancies, especially in children. Therefore, therapeutic options are limited, and few standardized therapy regimens exist.

Case presentation: We report a case of a 14-year-old white girl. In 2011 she was diagnosed with a metastasized, well-differentiated pancreatic neuroendocrine tumor with expression of synaptophysin and chromogranin A. We describe her clinical course with special attention to her individual therapeutic regimens while bringing together several disciplines of medicine.
\end{abstract}

Conclusions: In patients such as ours, surgical intervention may be the only therapy that will lead to long-term survival.

Keywords: Functioning pancreatic neuroendocrine tumor (F-PanNET), Islet cell tumors, Non-functioning pancreatic neuroendocrine tumor (NF-PanNET), Pancreatic neuroendocrine tumor (PanNET), Yttrium-90 DOTATOC therapy

\section{Introduction}

Metastasized pancreatic neuroendocrine tumors (PanNETs) are rare malignancies, especially in children and young adolescents, for which there is no standard therapeutic regimen. These lesions carry a poor prognosis. In this report, we present a case of a teenage girl (14-years old at age of first presentation) with a metastasized well-differentiated PanNET. A multidisciplinary therapeutic approach was designed by pediatric hematologists-oncologists, pediatric surgeons, visceral surgeons, pediatric radiologists and radio-oncologists. The primary tumor as well as its metastases have been kept in check to date. The patient is in good condition with little limitation of her daily activities.

\section{Case presentation}

In 2010, a 14-year-old white girl presented to our hospital and described having symptoms of ongoing abdominal pain of approximately 3 weeks' duration that was responsive to analgesic medication. She reported no prior history of nausea, vomiting, diarrhea, fever, sweating or weight

\footnotetext{
* Correspondence: tina.tremmel@med.uni-heidelberg.de

${ }^{1}$ Department of Pediatric Surgery, University Hospital Heidelberg, INF 110,

69120 Heidelberg, Germany

Full list of author information is available at the end of the article
}

loss. Her family history has been uneventful; both parents and a 19-year-old sister are healthy.

An abdominal ultrasound taken at that time showed normal upper abdominal organs and multiple enlarged lymph nodes that had previously been thought to be a reaction to a nonspecific infection. Her pain increased 2.5 weeks later, resulting in another visit to the emergency room. An abdominal magnetic resonance imaging (MRI) scan revealed a suspicious area in the head of her pancreas, persisting enlarged lymph nodes and unclear liver lesions (Fig. 1 and Fig. 2).

A scheduled surgical biopsy was taken after the patient's overall condition worsened. In addition to increasing pain, she lost appetite and weight. The histopathological results of the pancreas and liver examinations revealed a metastasized well-differentiated PanNET with expression of synaptophysin and chromogranin A (CgA). The outer membrane surface of the tumor cells expressed somatostatin receptor 2 (SSR2), and the active proliferative rate of Ki67 (a pathological grading marker) was 20 to $25 \%$ (Fig. 3). The results of additional bone marrow biopsies were unremarkable.

Extensive further diagnostic tests were done: laboratory testing and imaging with computed tomography (CT), ${ }^{68} \mathrm{Ga}$-DOTA-tyrosine octreotide $\left({ }^{68} \mathrm{Ga}\right.$-DOTATOC) positron emission tomography (PET)-CT, thoracic X-ray, 


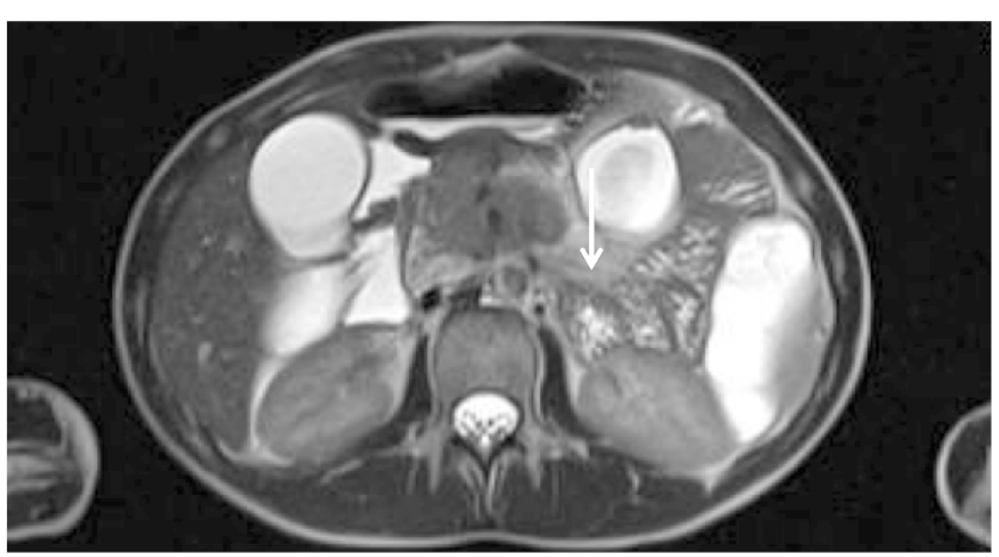

Fig. 1 Axial, T2-weighted, half-Fourier single-shot turbo spin-echo hydromagnetic resonance imaging scan of the patient's abdomen. Retroperitoneal tissue augmentation of the dorsal pancreas is visualized with contrast enhancement (arrow)

electrocardiography and echocardiography. Her blood work and urine analysis results are shown in Table 1.

In proximity to the previously described lesions, a widely spreading tumor with extensive surrounding of her superior mesenteric artery and her common hepatic artery was detected on imaging studies. Compression of

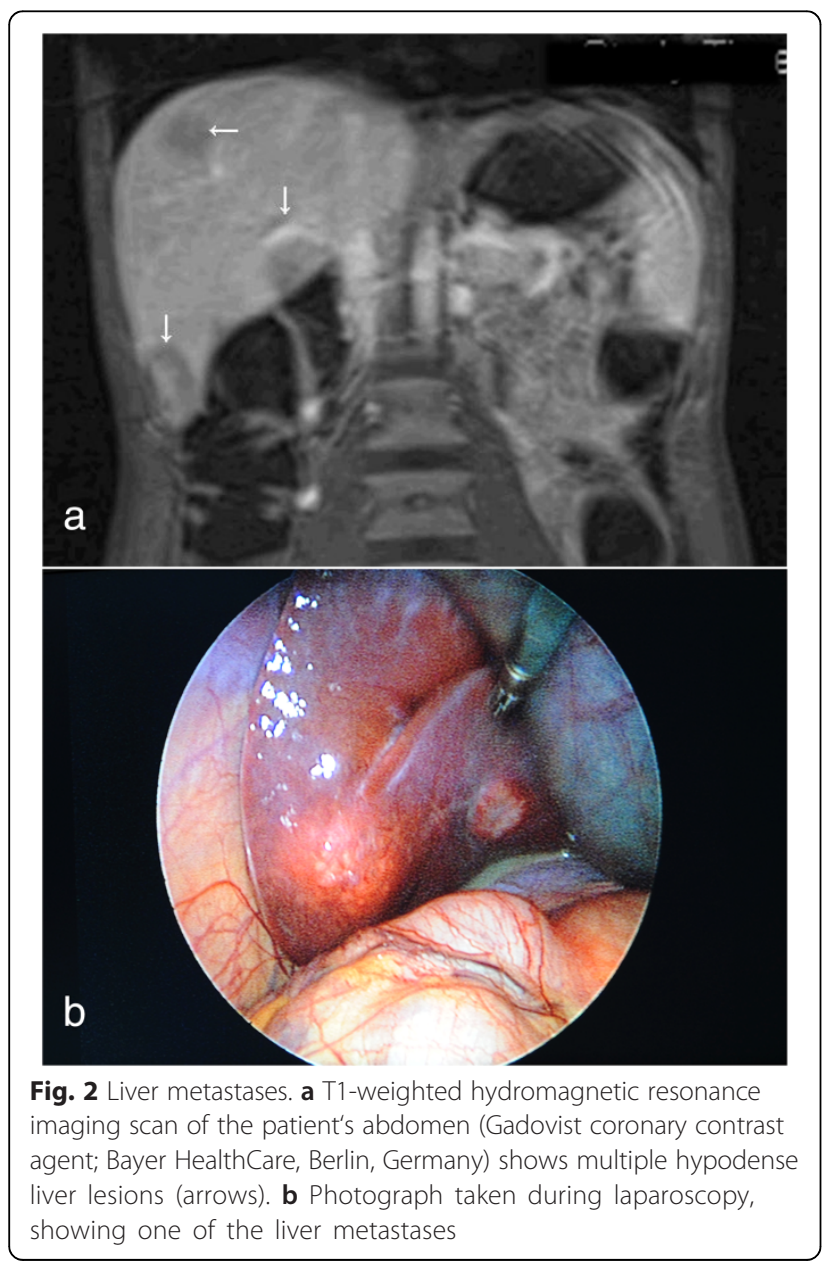

her splenic vein and her superior mesenteric vein, as well as obstruction of her portal vein with subsequent intestine vein insufficiency and cholestasis, was also diagnosed. These anatomic findings prompted us to refrain from a (primary) curative surgical approach because of possible life-threatening circumstances.

On the basis of these diagnostic test results, an interdisciplinary decision was made to downsize the tumor with an intra-arterial 4-GBq Lu-177/4-GBq yttrium (Y)-90-DOTATOC therapy in combination with an oral radiosensitizing chemotherapy with temozolomide (5 days) and thalidomide (4 days) as an individual approach 2 weeks later. Because of an expected aplasia and the main concern of weakening her overall condition, as well as to avoid endangering the planned extended surgical procedures, cisplatin and etoposide were not used.

Despite these interventions, her condition continued to worsen. Acute pancreatitis and biliary complications developed. Analgesic treatment failed to relieve her pain. As a result, surgery was indicated, despite the increased risk for potentially fatal complications.

Surgery with tumor resection took place in the same month. The following steps were performed: (1) extended pancreatic head resection, duodenectomy and cholecystectomy; (2) placement of a temporary GORETEX mesenteric-caval shunt and a temporary arterial aortohepatic shunt; (3) distal gastric resection with reconstruction with gastrojejunostomy (end to side), pancreaticojejunostomy and hepaticojejunostomy; (4) right hemicolectomy with terminal ileostomy and Hartmann procedure; (5) extended soft tissue resection, including the whole mesenteric root; (6) resection of the portal vein with vessel interpolation by the femoral superficial vein (from left adductor channel); (7) reconstruction of the hepatic artery with end-to-end anastomosis on the celiac trunk; and (8) atypical segmental liver resection of one metastasis (segment 2/3) (Fig. 4). 


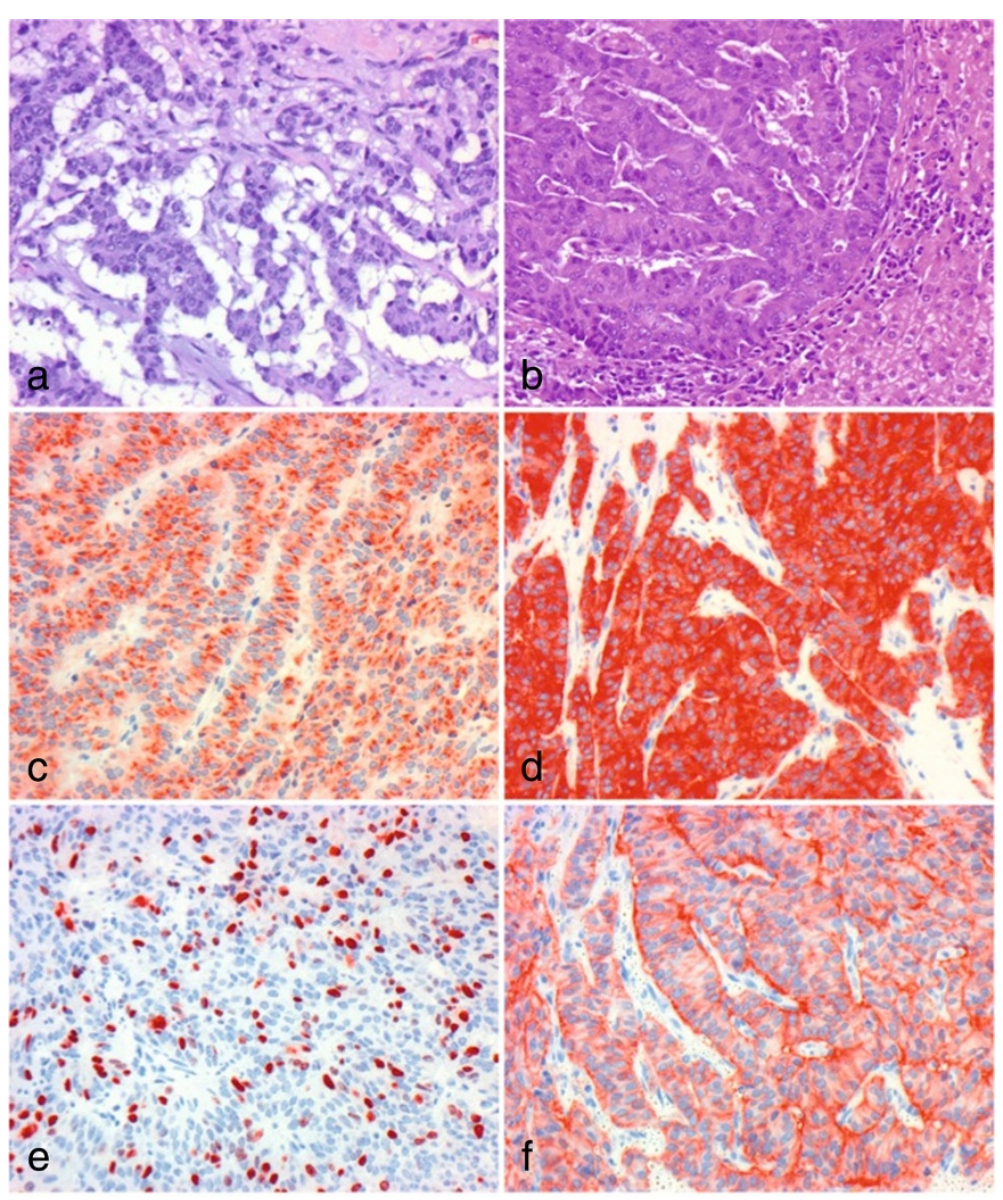

Fig. 3 Histopathological findings. Microscopic aspects of primary tumor (a) and liver metastasis (b through $\mathbf{f}$ ). As shown in hematoxylin and eosin-stained sections $(\mathbf{a}, \mathbf{b})$, the tumor displays an organoid growth pattern with predominant trabecular formations of atypical epithelial cells. Nuclei show only little to moderate pleomorphism, and the cytoplasm is well formed and sometimes contains vacuoles. On immunohistochemical examination, tumor cells show positivity for the neuroendocrine markers chromogranin A (c) and synaptophysin (d). Ki67 staining (e) reveals high proliferative activity. The tumor cells display membranous positivity of the somatostatin receptor 2a (f)

The patient recovered without major complications and could finally be dismissed with home parenteral nutrition 4 weeks later.

The first DOTATOC-PET scan obtained postoperatively showed two remaining small, right-sided liver metastases in segments 6 and 8 .

Six weeks later, a right hemihepatectomy without touching the primarily made biliodigestive anastomosis was performed as planned, followed by another DOTATOC-PET scan 1 month later. We detected that the patient was having a good response to therapy without signs of residual tumor. However, splenomegaly with high DOTATOC uptake was diagnosed. Because of the importance of the nucleotide therapy with aspired further consolidating circles and the vanished option for intra-arterial application owing to the resective surgery, splenectomy was the only opportunity to guarantee successful intravenous DOTATOC therapy. Therefore, a splenectomy was performed. In the same procedure, the ileostomy was taken down.
The next intravenous Lu-177/DOTATOC therapy was done. In the same month $(6$ months after the histopathological specimen results), an oral tyrosine kinase inhibitor medication with sunitinib was initiated. A follow-up examination 2 months later with DOTATOCPET and MRI scans was unremarkable. The patient recovered in a rehabilitation unit without being dependent on parenteral nutrition any longer.

Unfortunately, the next follow up DOTATOC-PET scan taken 1 year later showed increased uptake in her para-aortal lymph nodes and left inguinal soft tissue, so metastases of the primary tumor were expected (Fig. 5). Surgical extirpation of the suspicious left-sided inguinal lesion confirmed this hypothesis. The same endocrine malignant tumor cells could be seen (Ki-67: 30\%; SSR2/ DOTATOC-positive). Consequently, a para-aortic lymphadenectomy was performed.

Afterward, the patient additionally received intravenous Y-90-DOTATOC therapy. Despite those interventions, the 
Table 1 Laboratory values

\begin{tabular}{lll}
\hline Test & Value & Normal range \\
\hline Glutamic oxaloacetic transaminase & $277 \mathrm{U} / \mathrm{L}$ & $<35 \mathrm{U} / \mathrm{L}$ \\
Glutamic pyruvic transaminase & $342 \mathrm{U} / \mathrm{L}$ & $<35 \mathrm{U} / \mathrm{L}$ \\
Alkaline phosphatase & $233 \mathrm{U} / \mathrm{L}$ & 55 to $105 \mathrm{U} / \mathrm{L}$ \\
y-Glutamyl transferase & $339 \mathrm{U} / \mathrm{L}$ & $<40 \mathrm{U} / \mathrm{L}$ \\
Total bilirubin & $0.7 \mathrm{mg} / \mathrm{dl}$ & $<1 \mathrm{mg} / \mathrm{dl}$ \\
Conjugated bilirubin & $0.26 \mathrm{mg} / \mathrm{dl}$ & $<0.3 \mathrm{mg} / \mathrm{dl}$ \\
Amylase & $81 \mathrm{U} / \mathrm{L}$ & 8 to $53 \mathrm{U} / \mathrm{L}$ \\
Lipase & $143 \mathrm{U} / \mathrm{L}$ & $<51 \mathrm{U} / \mathrm{L}$ \\
Urea & $2.7 \mathrm{mg} / \mathrm{dl}$ & $<6 \mathrm{mg} / \mathrm{dl}$ \\
CgA & $293.1 \mathrm{U} / \mathrm{ml}$ & $<25 \mathrm{U} / \mathrm{ml}$ \\
NSE & $26.5 \mu \mathrm{g} / \mathrm{L}$ & $<17 \mu \mathrm{g} / \mathrm{L}$ \\
$\beta$-hCG & $<1 \mathrm{UU} / \mathrm{ml}$ & $<5 \mathrm{U} / \mathrm{ml}$ \\
AFP & $<1 \mathrm{U} / \mathrm{ml}$ & $<8 \mathrm{U} / \mathrm{ml}$ \\
Urine analysis & $\mathrm{Unremarkable}$ &
\end{tabular}

AFP, a-fetoprotein; $\beta$-hCG, Human chorionic gonadotropin, $\beta$-subunit; CgA, Chromogranin A; NSE, Neuron-specific enolase

next DOTATOC-PET scan still showed suspicious paraaortic lymph nodes. Repeat resection of the para-aortic lymph nodes was performed, followed by intravenous Ac225-DOTATOC therapy.

Owing to the stable oncologic situation and regarding the cumulative dose of radioactivity, with its increasing risk of chronic nephrotoxicity and bone marrow toxicity, no further nuclear medical therapy was planned as of this writing. Meanwhile, somatostatin (SST) analogue therapy with Somatuline Autogel ${ }^{\circ} 60 \mathrm{mg}$ (IPSEN, Signes, France), a long-lasting lanreotide option (injected subcutaneously once per month), as a non-radioactive blockade of tumor receptors was started to achieve remission.

The follow-up screening with DOTATOC-PET/CT 1.5 years after her first diagnosis indicated nonspecific nuclide uptake in her right breast and in one left-sided inguinal lymph node, as well as new high nuclide uptake in the right temporobasal part of her brain. Subsequent MRI of her brain confirmed a noticeable lesion raising strong suspicion of cranial metastases. Those lesions were irradiated with the patient in stereotactic positioning, and a good response was observed on the next MRI. In another surgical intervention, the left-sided inguinal lymph node was removed, which was pathologically confirmed as a metastasis of the primary tumor.

The patient reports that she is unaffected and repeat imaging is scheduled at short intervals to ensure regular follow-up.

The case presentation is summarized in Fig. 6 .

\section{Discussion}

PanNETs are rare pancreatic neoplasms compared with their more common exocrine counterparts. Arising from the pancreatic islets of Langerhans, they are also called islet cell tumors. They occur in 1 to 2 of 100,000 cases per year, accounting for less than $2 \%$ of all digestive malignant tumors and less than $1 \%$ of endocrine tumors. PanNETs represent approximately $3 \%$ of primary pancreatic neoplasms. Although they may manifest at any age, they most often occur in the fourth to sixth decades of life $[1,2]$.

Classically, PanNETs are divided into two groups: functioning pancreatic neuroendocrine tumors (F-Pan$\mathrm{NETs}$ ) and non-functioning pancreatic neuroendocrine tumors (NF-PanNETs). F-PanNETs are hormonally active and may lead to earlier diagnosis because of their hormone-related symptoms. Common types are insulinoma and gastrinoma, and rare types are VIPoma (vasoactive intestinal peptide), glucagonoma or somatostatinoma. For example, insulinomas often present with hypoglycemic symptoms and low blood glucose levels and are reversible after glucose intake; gastrinomas present with diarrhea, hypergastrinemia, gastric

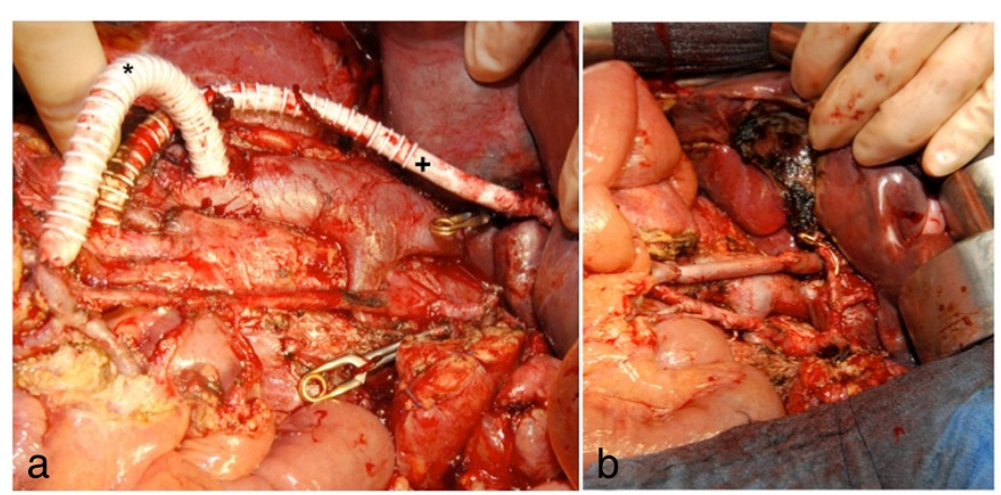

Fig. 4 Situs. a Intraoperative photograph of temporal placement of a mesenteric-caval GORE-TEX (*) shunt (GORE Medical, Flagstaff, AZ, USA) and a temporal arterial aortohepatic shunt $(+)$. $\mathbf{b}$ Intraoperative photograph taken after extended resection 


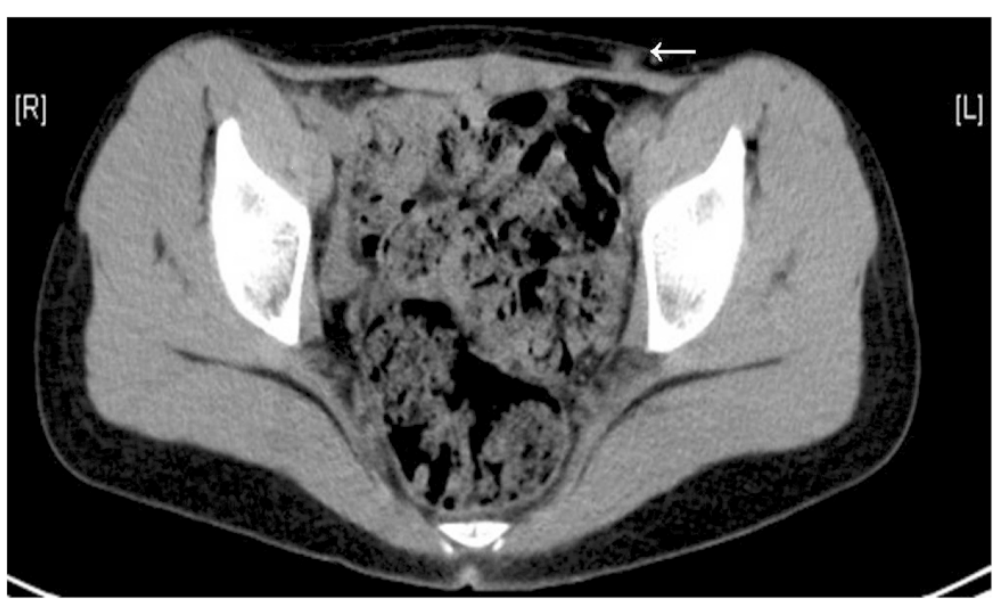

Fig. 5 Axial computed tomographic scan without contrast agent displaying left inguinal soft tissue. Suspicious hyperdense, irregularly shaped lesion (arrow) with a diameter of approximately $8 \mathrm{~mm}$ in the left inguinal soft tissue

acid hypersecretion and peptic ulcer diathesis, known as Zollinger-Ellison syndrome [2].

Although NF-PanNETs secrete a number of substances such as chromogranin, synaptophysin, neuronspecific enolase, pancreatic polypeptide and ghrelin, in contrast to F-PanNETs they do not present clinically with hormone-related symptoms. As a result, they often present later in the course of the disease with symptoms such as abdominal pain (35 to $75 \%$ ), weight loss (20 to $35 \%)$ and anorexia and nausea (45\%). Less frequent signs include obstructive jaundice (17 to 50\%), intraabdominal hemorrhage ( 4 to $20 \%$ ) or a palpable mass (7 to $40 \%$ ). Symptoms may also be attributable to metastatic disease, which occurs in, for example, the liver or bones (between 32 and $73 \%$ of cases are metastatic at diagnosis). NF-PanNETs may occur in association with the multiple endocrine neoplasia type 1 syndrome, von Hippel-Lindau syndrome, neurofibromatosis type 1 and tuberous sclerosis [2-4]. Genetic analyses were not performed in our patient. Nevertheless, considering the association of PanNETs with familial syndromes, this is an important issue, especially in young patients with PanNETs.
There are different possibilities for a diagnostic strategy: abdominal ultrasound, endoscopic ultrasound, abdominal CT, abdominal MRI, SST receptor scintigraphy, ${ }^{68} \mathrm{Ga}$-DOTATOC-PET/CT with a higher spatial resolution than scintigraphy, fine-needle aspiration cytology and/or biopsy, and laboratory tests with CgA $[5,6]$.

The 2010 World Health Organization classification system distinguishes between poorly differentiated neuroendocrine carcinomas (NECs) versus well-differentiated neuroendocrine tumors (NETs). All NECs are graded G3 with a Ki67 index greater than 20\%. NETs are divided into G2 grade with a Ki67 index of 3 to $20 \%$ and G1 grade with a Ki67 index of $2 \%$ or less. Most pancreatic nonfunctioning (NF) neuroendocrine neoplasms are well differentiated (that is, NETs); NF-NECs are uncommon [3].

In the present case, our patient has been diagnosed with a well-differentiated NF-NEC, despite a Ki67 index of 20 to $25 \%$, with a TNM classification of ypT3, ypN1(8/31), ypM1(liver) according to European Neuroendocrine Tumor Society consensus guidelines. Poorly differentiated pancreatic neuroendocrine neoplasms are characterized by their aggressive tumor biology, absence of SST receptors and poor prognosis.

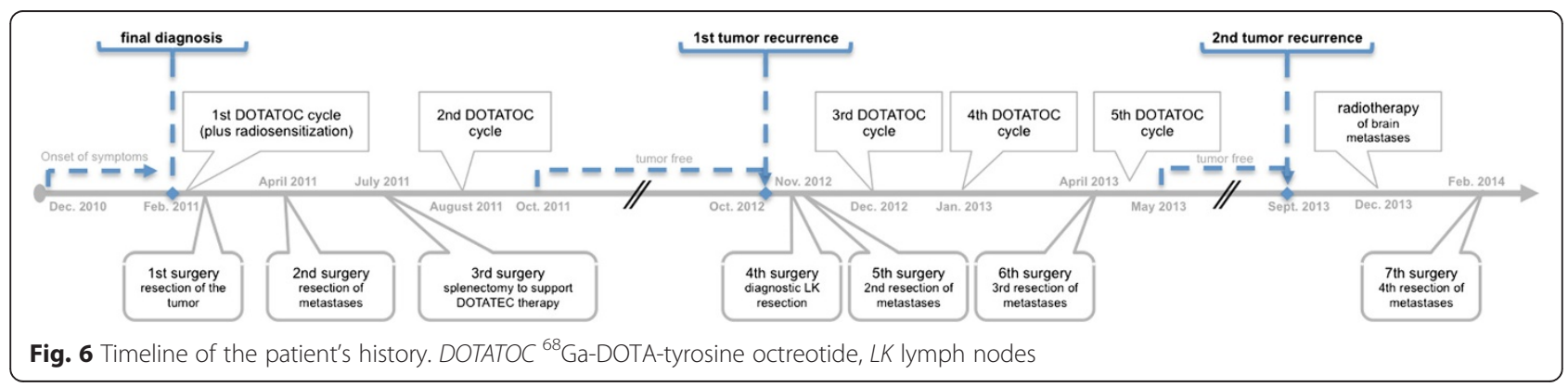


Clinical management involves a multidisciplinary approach, but surgery remains the only curative therapy for early-stage disease. It represents the treatment of choice in cases of localized PanNETs, including typical and atypical resections. In particular, radical resection of pancreatic tumors is associated with favorable long-term outcomes in children and adolescents [7]. Palliative surgery of locally advanced PanNETs is justified in selected patients. For patients with metastatic NF tumors, surgery of the primary tumor is recommended only for G1 and G2.

Further treatment options are SST analogues (for example, octreotide or lanreotide) for subgroups of patients with slowly progressive, low proliferative PanNETs; systemic chemotherapy (such as streptozotocin, 5fluorouracil or doxorubicin) in all PanNETs and NECs; peptide radionuclide receptor therapy (PRRT; SST analogues labeled with $\beta$-emitting radionuclides such as Y-90-DOTATOC) for patients with NETs with the presence of SST receptors; hepatic artery embolization or chemoembolization in patients with liver metastases who are not candidates for surgical resection; radiofrequency ablation in patients with unresectable metastases more than 5 to $7 \mathrm{~cm}$ in diameter; radiotherapy using high-energy isotope treatment for patients with inoperable disease; immunotherapy (for example, interferon, dendritic cell immunotherapy), which may be used to offer hormonerelated symptom control to patients in whom SST analogue therapy has failed; and molecular targeted therapy (including anti-epidermal growth factor receptor or anti-vascular endothelial growth factor receptor therapy such as sunitinib) for individual attempts and future options $[1,3,8]$.

In our patient, a primary curative surgical approach could not be used, owing to the anatomic findings of the spreading tumor. As the initial PRRT (with Y-90DOTATOC) combined with chemotherapy (temozolomide and thalidomide) failed [9], surgery convincingly emerged as the patient's last chance of potentially surviving. Successful treatments to that point of the patient's medical history involved different therapy options mainly combined repeated cycles of intravenous DOTATOC therapy with different surgical attempts, including resection of metastases and radiotherapy of the brain metastases. As shown, the patient's best chance of surviving is an individual approach combining all therapeutic options, and, in our patient, radical surgery was the most important part.

The median overall survival for patients with NFPanNETs is reportedly approximately 38 months. Patients with distant metastases have a median survival of 23 months, as compared with 70 to 124 months for those with localized disease [10-12]. Our patient is living and has a good quality of life approximately 42 months since the time of her first diagnosis.

\section{Conclusions}

PanNETs are rare tumors that often invade adjacent organs and anatomic structures, causing symptoms. Because of its rarity, randomized controlled studies have not been done and current treatment recommendations are based primarily on case series and individual treatment approaches. So far, total resection of the tumor is the treatment of choice and seems to be associated with the best prognosis, particularly when accompanied by further different therapies as mentioned above. Unresectable or partially resectable tumors and recurrent tumors can be treated with local and/or systemic radiation (PRRT) and/or adjuvant chemotherapy to downsize the tumor. Even then, surgery seems to be an important intervention option, which gives the patient relief from symptoms.

\section{Consent}

Written informed consent was obtained from the patient's legal guardian (and herself) for publication of this case report and accompanying images. A copy of the written consent is available for review by the Editor-inChief of this journal.

\section{Abbreviations \\ CT: Computed tomography; DOTATOC: DOTA-tyrosine octreotide; F-PanNET: Functioning pancreatic neuroendocrine tumor; MRI: Magnetic resonance imaging; NEC: Neuroendocrine carcinoma; NET: Neuroendocrine tumor; NF: Non-functioning; NF-PanNET: Non-functioning pancreatic neuroendocrine tumor; PanNET: Pancreatic neuroendocrine tumor; PET: Positron emission tomography; PRRT: Peptide receptor radionuclide therapy; SSR2: Somatostatin receptor 2; SST: Somatostatin; Y: Yttrium.}

\section{Competing interests}

The authors declare that they have no competing interests.

\section{Authors' contributions}

$\Pi$, SHC and PG analyzed and interpreted the patient data regarding the presented case and were major contributors in writing the manuscript. All authors read and approved the final manuscript.

\section{Acknowledgments}

The authors thank the patient and her family for their assistance and for providing consent for publication of this case report. The authors also thank the Institute of Pathology, University Hospital Heidelberg, for support of this study

\section{Author details}

${ }^{1}$ Department of Pediatric Surgery, University Hospital Heidelberg, INF 110, 69120 Heidelberg, Germany. ${ }^{2}$ Department of Pediatric Surgery, University Hospital Basel, Spitalstrasse 33, 4056 Basel, Switzerland.

Received: 4 February 2015 Accepted: 10 August 2015 Published online: 05 October 2015

\section{References}

1. Delaunoit T, Neczyporenko F, Rubin J, Erlichman C, Hobday TJ. Medical management of pancreatic neuroendocrine tumors. Am J Gastroenterol. 2008;103:475-83.

2. Ehehalt F, Saeger HD, Schmidt CM, Grützmann R. Neuroendocrine tumors of the pancreas. Oncologist. 2009;14:456-67.

3. Falconi M, Bartsch DK, Eriksson B, Klöppel G, Lopes JM, O'Connor JM, et al. ENETS Consensus Guidelines for the management of patients with digestive 
neuroendocrine neoplasms of the digestive system: well-differentiated pancreatic non-functioning tumors. Neuroendocrinology. 2012;95:120-34

4. Gläsker S, Neumann HPH, Koch CA, Vortmeyer AO. Von Hippel-Lindau Syndrome. In: De Groot LJ, Beck-Peccoz P, Chrousos G, Dungan K, Grossman A, Hershman JM, Koch C, McLachlan R, New M, Rebar R, Singer F, Vinik A, Weickert MO, editors. Endotext. South Dartmouth: MDText.com; 2012.

5. Howell DL, O'Dorisio MS. Management of neuroendocrine tumors in children, adolescents, and young adults. J Pediatr Hematol Oncol. 2012;34 Suppl 2:S64-68.

6. Lachter J, Arkovitz MS, Postovski S, Waldner JM, Shaoul R, Ishay OB, et al. EUS-FNA for a pancreatic neuroendocrine tumor in a four-year-old daughter of a woman exposed to radiation at Chernobyl. Case Rep Gastrointest Med. 2012;2012:462139.

7. Marchegiani G, Crippa S, Malleo G, Partelli S, Capelli P, Pederzoli P, et al. Surgical treatment of pancreatic tumors in childhood and adolescence: uncommon neoplasms with favorable outcome. Pancreatology. 2011;11:383-9.

8. Grozinsky-Glasberg S, Gross DJ. New drugs in the therapy of neuroendocrine tumors. J Endocrinol Invest. 2012;35:930-6.

9. Kulke MH, Stuart K, Enzinger PC, Ryan DP, Clark JW, Muzikansky A, et al. Phase II study of temozolomide and thalidomide in patients with metastatic neuroendocrine tumors. J Clin Oncol. 2006;24:401-6.

10. Rojas Y, Warneke CL, Dhamne CA, Tsao K, Nuchtern JG, Lally KP, et al. Primary malignant pancreatic neoplasms in children and adolescents: a 20 year experience. J Pediatr Surg. 2012;47:2199-204.

11. Perez EA, Gutierrez JC, Koniaris LG, Neville HL, Thompson WR, Sola JE. Malignant pancreatic tumors: incidence and outcome in 58 pediatric patients. J Pediatr Surg. 2009;44:197-203.

12. National Cancer Institute. Surveillance, Epidemiology, and End Results (SEER) program. http://seer.cancer.gov/

\section{Submit your next manuscript to BioMed Central and take full advantage of:}

- Convenient online submission

- Thorough peer review

- No space constraints or color figure charges

- Immediate publication on acceptance

- Inclusion in PubMed, CAS, Scopus and Google Scholar

- Research which is freely available for redistribution 